\title{
Usefulness and safety of a dedicated team to prone patients with severe ARDS due to COVID-19
}

\author{
Antoine Kimmoun ${ }^{1}$, Bruno Levy $^{1 *} \mathbb{D}$, Bruno Chenuel $^{2}$ and The DV-Team group
}

\section{To the Editor:}

Prone position ventilation has been shown to improve oxygenation and survival in patients with severe acute respiratory distress syndrome (ARDS) [1]. Facing the coronavirus disease 2019 (COVID-19) pandemic, prone positioning (PP) is of crucial importance to treat severe ARDS patients [2]. Nevertheless, the high number of ICU admissions quickly overwhelmed the ability of the daily ICU team to place patients in $\mathrm{PP}$, a complex and time-consuming maneuver. Thus, we created a dedicated medical team with reassigned volunteers to cope with the large number of patients requiring $\mathrm{PP}$.

PP Team consisted of five volunteers: a senior medical non-intensivist physician placed at the patient's head to secure the endotracheal tube and four medical fellows or medical students placed at each side of the bed. For patients treated with VV-ECMO, a supplementary physician was added to secure the lines. Since PP is a complex procedure and has many potential adverse events requiring adequate and well-trained staff, volunteers received previously a theoretical training and a hands-on ad hoc training session. PP teams followed the guidelines for PP placement [1].

This retrospective observational study was performed in our extended ICU (from 22 to 46 beds), from the first day of deployment of PPT (March 23 to April 23, 2020).

The main characteristics and outcomes of prone positioned patients $(n=63)$ are presented in Table 1 .
A total of 367 placements in a prone or supine position were performed during the 1-month study period (Table 2).

This specific medical team of trained nonintensivist volunteers was able to manage this delicate PP task without any major adverse events such as cardiac arrest or unscheduled extubation when compared to the relatively high incidence (respectively 6.8 and $13.3 \%$ ) observed in Guérin et al. study [1]. Our studied population was comparable to already published series of severe ARDS, and we found a similar mortality (26\%) despite a lower initial P/F ratio and COVID-19 association [1]. Interestingly, we recorded a greater survival rate than reported by Richardson et al. in the New York area at their edge of COVID-19 pandemic, but they did not detail the use of PP [3].

This innovative management allowed three major benefits: (i) critical relief of permanent intensive care team's workload; (ii) reduction of the nurse-to-patient ratio, permitting also the reassignment of critical care nurses to newly created ICUs; and (iii) devoid of any self-censorship for fear of overwork and burn-out, intensivist physicians were able to strictly follow PP guideline recommendations, ensuring the best standard of care for ARDS patients.

Since the pathophysiology is poorly understood $[4,5]$, the specific role of PP among the optimal management for COVID-19 patients with ARDS, in order to reduce mortality needs to be addressed.

\footnotetext{
* Correspondence: blevy5463@gmail.com

'CHRU-Nancy, INSERM U1116, Médecine Intensive et Réanimation Brabois, Université de Lorraine, F-54000 Nancy, France

Full list of author information is available at the end of the article
} 
Table 1 Initial characteristics and outcome of the prone positioned ARDS COVID-19 population (data are expressed as median (interquartile range, IQR) or number (\%) as appropriate. After visual assumption of normality, Wilcoxon rank tests were applied for continuous variables. For categorical variables, Fisher's exact or chi ${ }^{2}$ tests were applied as appropriate)

\begin{tabular}{|c|c|c|c|c|c|c|c|}
\hline Variables & $\begin{array}{l}n, \text { total } \\
\text { available }\end{array}$ & $\begin{array}{l}\text { Total, } \boldsymbol{n}(\%) \text { or } \\
\text { median (IQR) } \\
(\boldsymbol{n}=63)\end{array}$ & $\begin{array}{l}\boldsymbol{n} \text {, available for } \\
\text { ICU survivors }(\boldsymbol{n}= \\
46){ }^{*}\end{array}$ & $\begin{array}{l}\text { ICU survivors, } \\
\boldsymbol{n}(\%) \text { or median } \\
\left(\text { IQR) }{ }^{*}\right.\end{array}$ & $\begin{array}{l}\boldsymbol{n} \text {, available for ICU } \\
\text { non-survivors }(\boldsymbol{n}= \\
16)^{*}\end{array}$ & $\begin{array}{l}\text { ICU non-survivors, } \\
\boldsymbol{n}(\%) \text { or median } \\
(\mathrm{IQR}) *\end{array}$ & $p$ \\
\hline \multicolumn{8}{|l|}{ Demographic data } \\
\hline Female ratio & 63 & 15 (24\%) & 46 & $10(22 \%)$ & 16 & $5(31 \%)$ & 0.50 \\
\hline Age (years) & 63 & $64(56-70)$ & 46 & $62(54-69)$ & 16 & $67(64-74)$ & 0.045 \\
\hline Weight (kg) & 61 & $89(75-103)$ & 45 & $90(80-103)$ & 15 & $89(73-106)$ & 0.68 \\
\hline Body mass index $\left(\mathrm{kg} / \mathrm{m}^{2}\right)$ & 61 & $30(25-36)$ & 45 & $30(26-35)$ & 15 & $29(25-46)$ & 0.87 \\
\hline SAPS ॥ & 63 & $42(31-57)$ & 46 & $37(27-57)$ & 16 & $46(42-59)$ & 0.030 \\
\hline \multicolumn{8}{|l|}{ Medical history } \\
\hline Diabetes mellitus & 63 & 17 (27\%) & 46 & $11(24 \%)$ & 16 & $5(31 \%)$ & 0.74 \\
\hline Hypertension & 63 & 30 (48\%) & 46 & $19(41 \%)$ & 16 & $10(62 \%)$ & 0.14 \\
\hline Chronic respiratory disease & 63 & $16(25 \%)$ & 46 & $13(28 \%)$ & 16 & $3(19 \%)$ & 0.53 \\
\hline $\begin{array}{l}\text { Chronic } \\
\text { immunosuppressiont }\end{array}$ & 63 & $5(8 \%)$ & 46 & $2(4 \%)$ & 16 & $3(19 \%)$ & 0.10 \\
\hline $\begin{array}{l}\text { Chronic Cardiovascular } \\
\text { disease }\end{array}$ & 63 & $16(25 \%)$ & 46 & $12(26 \%)$ & 16 & $3(19 \%)$ & 0.74 \\
\hline Chronic kidney disease & 63 & $3(5 \%)$ & 46 & $2(4 \%)$ & 16 & $1(6 \%)$ & 1.00 \\
\hline \multicolumn{8}{|l|}{ Respiratory parameters } \\
\hline $\begin{array}{l}\text { Static compliance ( } \mathrm{ml} / \\
\mathrm{CmH}_{2} \mathrm{O} \text { ) before first prone } \\
\text { positioning }\end{array}$ & 46 & $33(23-42)$ & 35 & $35(27-44)$ & 11 & $22(18-36)$ & 0.036 \\
\hline $\begin{array}{l}\mathrm{PaO}_{2} / \mathrm{F}_{1} \mathrm{O}_{2} \text { ratio before first } \\
\text { prone positioning }\end{array}$ & 63 & $92(70-117)$ & 46 & $96(70-120)$ & 16 & $86(64-111)$ & 0.54 \\
\hline $\begin{array}{l}\text { Number of prone } \\
\text { positioning per patient }\end{array}$ & 63 & $3(2-6)$ & 46 & $3(2-6)$ & 16 & $4(3-8)$ & 0.19 \\
\hline \multicolumn{8}{|l|}{ Events in ICU } \\
\hline Vasopressors administered & 63 & 38 (60\%) & 46 & $23(50 \%)$ & 16 & $14(88 \%)$ & 0.008 \\
\hline W-ECMO & 63 & $14(22 \%)$ & 46 & $11(24 \%)$ & 16 & $3(19 \%)$ & 1.00 \\
\hline Renal replacement therapy & 63 & $8(13 \%)$ & 46 & $4(9 \%)$ & 16 & $4(25 \%)$ & 0.19 \\
\hline ICU length of stay (days) & 62 & $19(14-31)$ & 46 & $20(15-32)$ & 16 & $16(12-28)$ & 0.24 \\
\hline
\end{tabular}

*1 patient still in ICU

${ }^{\dagger}$ Representing active cancer medical history or chronic immunosuppressor therapies 
Table 2 Prone and supine placements and reported adverse events during the procedure on the study period. (data are expressed as mean \pm standard deviation or number (percentage)). The 367 placements represent the placement in prone or supine positions

\begin{tabular}{ll}
\hline & Prone/Supine positioning placements, $\boldsymbol{n}(\%)$ or mean \pm SD $(\boldsymbol{n}=367)$ \\
\hline Number of placements performed & $11.5 \pm 3.4$ \\
Daily & $7 \pm 1.4$ \\
First 2-day period & $20 \pm 4.2$ \\
Acme 2-day period & $5 \pm 0$ \\
Last 2-day period & $124(34 \%)$ \\
Under W-ECMO & \\
Adverse events recorded during placements & \\
Major & 0 \\
$\quad$ Cardiac arrest & 0 \\
Unscheduled extubation & $5(1 \%)$ \\
Severe desaturation (SpO & $<85 \%)^{*}$ \\
Minor & $6(2 \%)$ \\
Accidental device removing or disconnectiont &
\end{tabular}

*Needing medical intervention

†Minor: one epistaxis following accidental removing of naso-gastric tube, four incidental disconnections of ventilator lines, one incidental removing of central venous catheter

\begin{abstract}
Acknowledgements
The DV-Team group

Collaborators:

S. Barde ${ }^{1}$, A. Didelot ${ }^{1}$, B. Chenuel ${ }^{1}$, P. Zieminski ${ }^{2}$, M. Lorcin ${ }^{3}$, C. Schweitzer ${ }^{3}$, R. Karunna ${ }^{4}$, V. Moulin ${ }^{3}$, M. Huet ${ }^{3}$, F. Lacour ${ }^{3}$, P. Rodriguez ${ }^{3}$, D. Grandmougin ${ }^{5}$, Y.

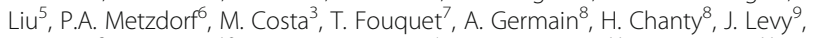
A. Didier ${ }^{8}$, J. Lawton ${ }^{10}$, C. Parietti-Winkler ${ }^{4}$, A. Manuguerra ${ }^{11}$, C. Mazeaud ${ }^{11}, C$. Gaulier $^{7}$, C. Rumeau ${ }^{4}$, L. Bourson ${ }^{10}, M$. Cholley-Roulleau ${ }^{12}$, A. Ionescu ${ }^{5}$, A. Gatin $^{13}$, M. Perez ${ }^{7}$, A. Schwanké ${ }^{7}$, G. Lauria $^{5}$, L. Freysz ${ }^{6}$, T. Cuinet $^{14}$, J. Chauvelot $^{4}$, C. Mottola ${ }^{15}$, T. Toussaint ${ }^{3}$, L. Dechambenoit ${ }^{3}$, F. Lagrange $^{11}$, C. Mathieu ${ }^{3}$, C. Clément ${ }^{3}$, H. Benamron ${ }^{4}$, L. Dubouis ${ }^{16}$, H. Kremer $^{17}$, L. Cabanel ${ }^{18}$, M. Falcetta $^{3}$, V. Gorzkowski ${ }^{4}$, P. Campoli ${ }^{16}$, J. Cavailhes $^{17}$, J. Zavoli ${ }^{19}$, C. Nominé-Criqui $^{7}$, J. Felloni ${ }^{20}$, V. Cloché-Fouquet ${ }^{21}$, F.G. Midon ${ }^{17}$, G. Vaz $^{17}$, D. Valentin ${ }^{3}$, V.

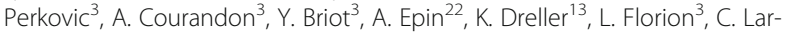
ose $^{3}$, M. Barron ${ }^{23}$, C. Sadoul ${ }^{24}$, M. Gimbert ${ }^{25}$, M. Fernandez ${ }^{17}$, T. Thomas ${ }^{3}$, P. Bichet $^{3}$, N. Petkunaite ${ }^{25}$, S. Brahami ${ }^{4}$, D. Nguyen ${ }^{4}$, G. Vaz $^{17}$, A. Schaefer ${ }^{13}, C$. Fabbri $^{3}$, C. Ferri ${ }^{17}$, A. Gegout ${ }^{3}$, A. Poncy ${ }^{19}$, R. Delaplace ${ }^{26}$, M. Ammisaid $^{5}$, J. Rebois $^{4}$, B. Vendeville ${ }^{3}$, C. Dubroux ${ }^{21}$, R. Raynaud ${ }^{11}$, S. Moog ${ }^{3}$, C. Cottez, L. Woirhaye $^{3}$, J. Menet ${ }^{21}$, A.C. Madkaud ${ }^{21}$, L. Naisseline ${ }^{3}$, C. Mathieu $^{3}$, T. Raze ${ }^{3}$, F. Violon ${ }^{27}$, M. Meiers ${ }^{17}$, D. Albanesi ${ }^{3}$, O. Durand ${ }^{3}$, L. Textoris $^{28}$, T Dubost $^{28}$ 1- Université de Lorraine, CHRU-Nancy, Centre Universitaire de Médecine du Sport et Activité Physique Adaptée, F-54000 Nancy, France.

2- Université de Lorraine, CHRU-Nancy, Médecine Vasculaire F-54000 Nancy, France.

3- Université de Lorraine, Faculté de Médecine de Nancy, CHRU-Nancy, F54000 Nancy, France.

4- Université de Lorraine, CHRU-Nancy, ORL et Chirurgie Cervico-Faciale, F54000 Nancy, France.

5- Université de Lorraine, CHRU-Nancy, Chirurgie Cardio-Vasculaire et Transplantations, F-54000 Nancy, France.

6- Université de Lorraine, CHRU-Nancy, Cardiologie Médicale, F-54000 Nancy, France.

7- Université de Lorraine, CHRU-Nancy, Unité Médicochirurgicale de Chirurgie Viscérale et Cancérologie, F-54000 Nancy, France.

8- Université de Lorraine, CHRU-Nancy, Chirurgie Digestive et Générale, F54000 Nancy, France.

9- Université de Lorraine, CHRU-Nancy, Biologie Médicale, F-54000 Nancy, France.

10- Université de Lorraine, CHRU-Nancy, Hépato-Gastro-Entérologie, F-54000 Nancy, France.
\end{abstract}

11- Université de Lorraine, CHRU-Nancy, Urologie, F-54000 Nancy, France.
12- Université de Lorraine, CHRU-Nancy, Chirurgie de la main, F-54000 Nancy, France.

13- Université de Lorraine, CHRU-Nancy, Pédiatrie, F-54000 Nancy, France.

14- Université de Lorraine, CHRU-Nancy, Chirurgie Orthopédique Infantile, F54000 Nancy, France.

15- Université de Lorraine, CHRU-Nancy, Néphrologie, F-54000 Nancy, France. 16- Université de Lorraine, CHRU-Nancy, Anatomopathologie, F-54000 Nancy, France.

17- Université de Lorraine, CHRU-Nancy, Chirurgie Orthopédique, F-54000 Nancy, France.

18- Université de Lorraine, CHRU-Nancy, Gynécologie Obstétrique, F-54000 Nancy, France.

19- Université de Lorraine, CHRU-Nancy, Département de Médecine Générale, F-54000 Nancy, France.

20- Université de Lorraine, CHRU-Nancy, Rééducation Fonctionnelle, F-54000 Nancy, France.

21- Université de Lorraine, CHRU-Nancy, Ophtalmologie, F-54000 Nancy, France.

22- Université de Lorraine, CHRU-Nancy, Nutrition. F-54000 Nancy, France. 23- Université de Lorraine, CHRU-Nancy, Chirurgie Maxillo-Faciale et Stomatologique, F-54000 Nancy, France.

24- Université de Lorraine, CHRU-Nancy, Chirurgie Vasculaire et Endoluminale, F-54000 Nancy, France.

25- Université de Lorraine, CHRU-Nancy, Consultations Pathologies Professionnelles. F-54000 Nancy, France.

26- Université de Lorraine, CHRU-Nancy, Chirurgie Infantile Viscérale. F-54000 Nancy, France.

27- Université de Lorraine, CHRU-Nancy, Médecine Nucléaire. F-54000 Nancy, France.

28- Université de Lorraine, CHRU-Nancy, INSERM U1116, Médecine Intensive et Réanimation Brabois, F-54000 Nancy, France

\section{Authors' contributions}

Data acquisition: AK. Data analysis: AK. Data interpretation: all authors. Manuscript drafting and revising: all authors. The authors read and approved the final manuscript.

Funding

There was no financial funding. 


\section{Availability of data and materials}

All data generated or analyzed during this study are included in this published article. The data used to support the findings of this study are available from the corresponding author upon request.

\section{Ethics approval and consent to participate}

Not applicable.

\section{Consent for publication}

Not applicable.

\section{Competing interests}

The authors declare that they have no competing interests related to the present publication.

\section{Author details}

${ }^{1}$ CHRU-Nancy, INSERM U1116, Médecine Intensive et Réanimation Brabois, Université de Lorraine, F-54000 Nancy, France. ${ }^{2} \mathrm{CHRU}-\mathrm{Nancy}$, Centre Universitaire de Médecine du Sport et Activité Physique Adaptée, Explorations Fonctionnelles Respiratoires, EA DevAH, Département de Physiologie, Université de Lorraine, F-54000 Nancy, France. ${ }^{3} \mathrm{CHRU}-\mathrm{Nancy}$, Centre Universitaire de Médecine du Sport et Activité Physique Adaptée, Université de Lorraine, F-54000 Nancy, France. ${ }^{4} \mathrm{CHRU-Nancy,} \mathrm{Médecine}$ Vasculaire, Université de Lorraine, F-54000 Nancy, France. ${ }^{5}$ Faculté de Médecine de Nancy, CHRU-Nancy, Université de Lorraine, F-54000 Nancy, France. ${ }^{6} \mathrm{CHRU}-\mathrm{Nancy}$, ORL et Chirurgie Cervico-Faciale, Université de Lorraine, F-54000 Nancy, France. ${ }^{7} \mathrm{CHRU}-\mathrm{Nancy}$, Chirurgie Cardio-Vasculaire et Transplantations, Université de Lorraine, F-54000 Nancy, France. ${ }^{8} \mathrm{CHRU}-\mathrm{Nancy}$, Cardiologie Médicale, Université de Lorraine, F-54000 Nancy, France. ${ }^{9} \mathrm{CHRU}$-Nancy, Unité Médicochirurgicale de Chirurgie Viscérale et Cancérologie, Université de Lorraine, F-54000 Nancy, France. ${ }^{10} \mathrm{CHRU-Nancy,}$ Chirurgie Digestive et Générale, Université de Lorraine, F-54000 Nancy, France. ${ }^{11} \mathrm{CHRU}-\mathrm{Nancy}$, Biologie Médicale, Université de Lorraine, F-54000 Nancy, France. ${ }^{12} \mathrm{CHRU}$-Nancy, Hépato-Gastro-Entérologie, Université de Lorraine, F-54000 Nancy, France. ${ }^{13} \mathrm{CHRU}-$ Nancy, Urologie, Université de Lorraine, F-54000 Nancy, France. ${ }^{14} \mathrm{CHRU}-$ Nancy, Chirurgie de la main, Université de Lorraine, F-54000 Nancy, France. ${ }^{15} \mathrm{CHRU}-$ Nancy, Pédiatrie, Université de Lorraine, F-54000 Nancy, France. ${ }^{16} \mathrm{CHRU}-N a n c y$, Chirurgie Orthopédique Infantile, Université de Lorraine, F-54000 Nancy, France. ${ }^{17} \mathrm{CHRU}$-Nancy, Néphrologie, Université de Lorraine, F-54000 Nancy, France. ${ }^{18} \mathrm{CHRU}-\mathrm{Nancy}$, Anatomopathologie, Université de Lorraine, F-54000 Nancy, France. ${ }^{19} \mathrm{CHRU}-$ Nancy, Chirurgie Orthopédique, Université de Lorraine, F-54000 Nancy, France. ${ }^{20} \mathrm{CHRU-Nancy,} \mathrm{Gynécologie} \mathrm{Obstétrique,} \mathrm{Université}$ de Lorraine, F-54000 Nancy, France. ${ }^{21}$ CHRU-Nancy, Département de Médecine Générale, Université de Lorraine, F-54000 Nancy, France. ${ }^{22} \mathrm{CHRU}$-Nancy, Rééducation Fonctionnelle, Université de Lorraine, F-54000 Nancy, France. ${ }^{23} \mathrm{CHRU}$-Nancy, Ophtalmologie, Université de Lorraine, F-54000 Nancy, France. ${ }^{24} \mathrm{CHRU-Nancy,} \mathrm{Nutrition,} \mathrm{Université} \mathrm{de} \mathrm{Lorraine,}$ F-54000 Nancy, France. ${ }^{25} \mathrm{CHRU}$-Nancy, Chirurgie Maxillo-Faciale et Stomatologique, Université de Lorraine, F-54000 Nancy, France.

${ }^{26} \mathrm{CHRU}-\mathrm{Nancy}$, Chirurgie Vasculaire et Endoluminale, Université de Lorraine, F-54000 Nancy, France. ${ }^{27} \mathrm{CHRU}$-Nancy, Consultations Pathologies Professionnelles, Université de Lorraine, F-54000 Nancy, France.

${ }^{28} \mathrm{CHRU}$-Nancy, Chirurgie Infantile Viscérale, Université de Lorraine, F-54000 Nancy, France. ${ }^{29} \mathrm{CHRU}$-Nancy, Médecine Nucléaire, Université de Lorraine, F-54000 Nancy, France. ${ }^{30}$ CHRU-Nancy, INSERM U1116, Médecine Intensive et Réanimation Brabois, Université de Lorraine, F-54000 Nancy, France.

Received: 2 June 2020 Accepted: 1 July 2020

Published online: 18 August 2020

\section{References}

1. Guérin C, Reignier J, Richard JC, Beuret P, Gacouin A, Boulain T, et al. Prone positioning in severe acute respiratory distress syndrome. N Engl J Med. 2013;368:2159-68.

2. Carsetti A, Damia Paciarini A, Marini B, et al. Prolonged prone position ventilation for SARS-CoV-2 patients is feasible and effective. Crit Care. 2020; 24.225

3. Richardson S, Hirsch JS, Narasimhan M, Crawford JM, McGinn T, Davidson KW, the Northwell COVID-19 Research Consortium. Presenting characteristics, comorbidities, and outcomes among 5700 patients hospitalized with COVID-19 in the New York city area. JAMA. 2020. https:// doi.org/10.1001/jama.2020.6775.

4. Gattinoni L, Chiumello D, Caironi P, Busana M, Romitti F, Brazzi L, et al. COVID-19 pneumonia: different respiratory treatments for different phenotypes? Intensive Care Med. 2020;46:1099-1102. https://doi.org/10. 1007/s00134-020-06033-2.

5. Gattinoni L, Chiumello D, Rossi S. COVID-19 pneumonia: ARDS or not? Crit Care. 2020;24:154. https://doi.org/10.1186/s13054-020-02880-z.

\section{Publisher's Note}

Springer Nature remains neutral with regard to jurisdictional claims in published maps and institutional affiliations.

\section{Ready to submit your research? Choose BMC and benefit from:}

- fast, convenient online submission

- thorough peer review by experienced researchers in your field

- rapid publication on acceptance

- support for research data, including large and complex data types

- gold Open Access which fosters wider collaboration and increased citations

- maximum visibility for your research: over $100 \mathrm{M}$ website views per year

At BMC, research is always in progress.

Learn more biomedcentral.com/submissions 